BMC

Evolutionary Biology

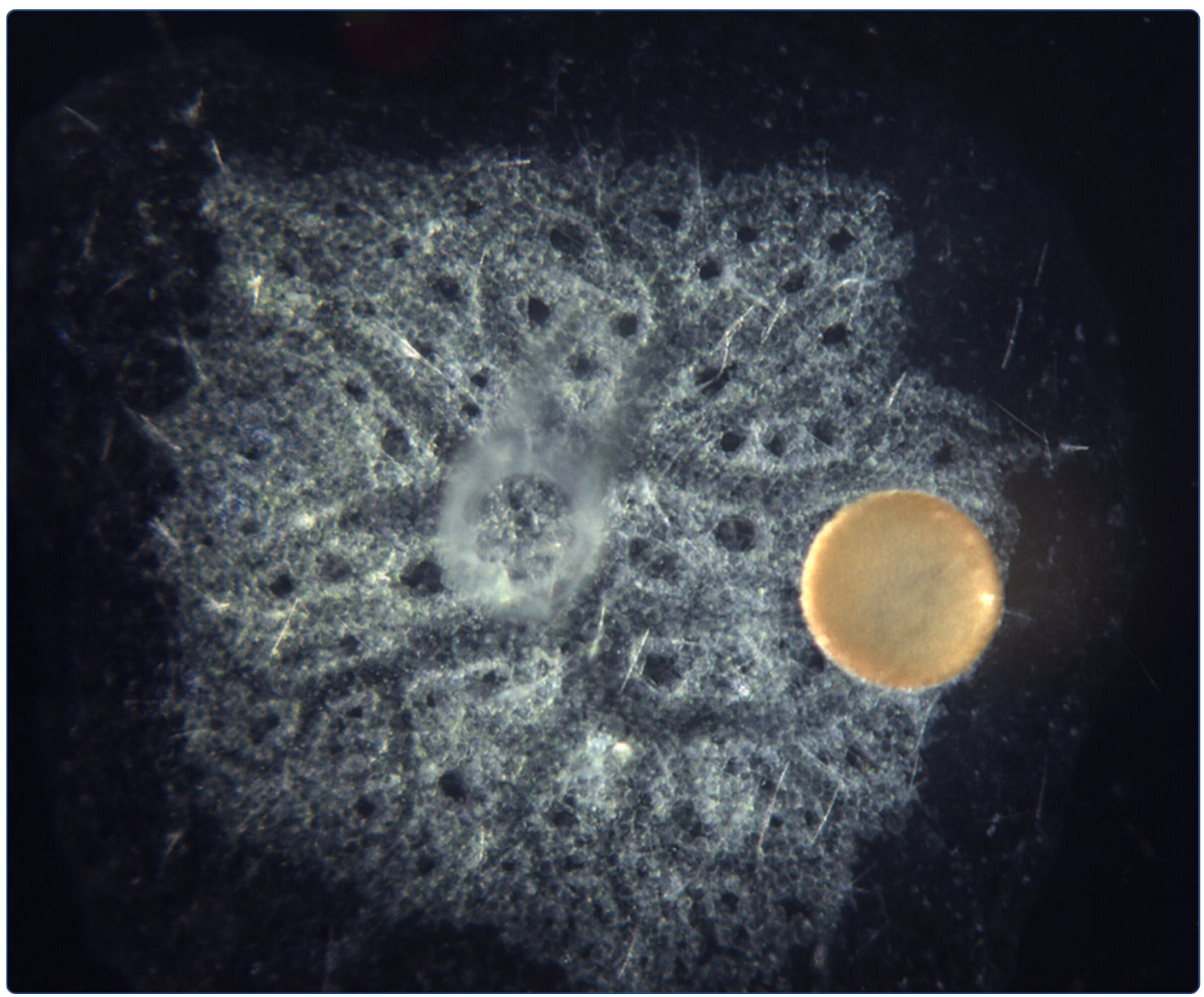

Evolutionary origins of sensation in metazoans: functional evidence for a new sensory organ in sponges

Ludeman et al.

( ) Biomed Central 


\title{
Evolutionary origins of sensation in metazoans: functional evidence for a new sensory organ in sponges
}

Danielle A Ludeman ${ }^{1}$, Nathan Farrar ${ }^{1}$, Ana Riesgo $^{2}$, Jordi Paps ${ }^{3}$ and Sally P Leys ${ }^{1 *}$

\begin{abstract}
Background: One of the hallmarks of multicellular organisms is the ability of their cells to trigger responses to the environment in a coordinated manner. In recent years primary cilia have been shown to be present as 'antennae' on almost all animal cells, and are involved in cell-to-cell signaling in development and tissue homeostasis; how this sophisticated sensory system arose has been little-studied and its evolution is key to understanding how sensation arose in the Animal Kingdom. Sponges (Porifera), one of the earliest evolving phyla, lack conventional muscles and nerves and yet sense and respond to changes in their fluid environment. Here we demonstrate the presence of non-motile cilia in sponges and studied their role as flow sensors.
\end{abstract}

Results: Demosponges excrete wastes from their body with a stereotypic series of whole-body contractions using a structure called the osculum to regulate the water-flow through the body. In this study we show that short cilia line the inner epithelium of the sponge osculum. Ultrastructure of the cilia shows an absence of a central pair of microtubules and high speed imaging shows they are non-motile, suggesting they are not involved in generating flow. In other animals non-motile, 'primary', cilia are involved in sensation. Here we show that molecules known to block cationic ion channels in primary cilia and which inhibit sensory function in other organisms reduce or eliminate sponge contractions. Removal of the cilia using chloral hydrate, or removal of the whole osculum, also stops the contractions; in all instances the effect is reversible, suggesting that the cilia are involved in sensation. An analysis of sponge transcriptomes shows the presence of several transient receptor potential (TRP) channels including PKD channels known to be involved in sensing changes in flow in other animals. Together these data suggest that cilia in sponge oscula are involved in flow sensation and coordination of simple behaviour.

Conclusions: This is the first evidence of arrays of non-motile cilia in sponge oscula. Our findings provide support for the hypothesis that the cilia are sensory, and if true, the osculum may be considered a sensory organ that is used to coordinate whole animal responses in sponges. Arrays of primary cilia like these could represent the first step in the evolution of sensory and coordination systems in metazoans.

Keywords: Porifera, Primary cilia, Evolution of nervous systems, Sensory systems, PKD

\section{Background}

Sensory systems use specialized cells or organelles to receive signals that are conducted through the body electrically or chemically [1]. Signal transduction in many unicellular eukaryotes occurs via cilia, which often have both motile and sensory roles [2-4]. The evolution of multicellularity necessarily involved the ability to transduce

\footnotetext{
*Correspondence: sleys@ualberta.ca

${ }^{1}$ Department of Biological Sciences, University of Alberta, CW 405 Biological

Sciences Building, Edmonton, Alberta T6G 2E9, Canada

Full list of author information is available at the end of the article
}

signals over longer distances, which in animals is now done by nerves [5] to allow rapid coordinated movements of the whole organism [6]. Although cilia play an important role in sensing the environment in both unicellular and multicellular animals, the evolutionary relationship of sensory cilia in unicellular eukaryotes, fungi and metazoans is unclear. Studies of sensory systems in the earliest evolving metazoans could shed light on shared common mechanisms of sensation.

Sponges lack a nervous system and while they are usually considered representatives of the first multicellular animals

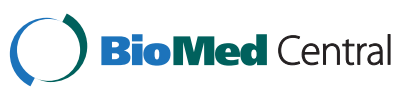


[7-10], some recent phylogenomic analyses place ctenophores more basally $[11,12]$ calling into question our understanding of the evolution of nerves and the ancestral metazoan state. Analysis of sponge genomes and transcriptomes has revealed a complex assortment of signaling molecules and proteins necessary for a post-synaptic scaffold $[13,14]$. Together with physiological evidence that glutamatergic signaling occurs in sponges $[15,16]$ this suggests that a signaling system similar to that seen in other metazoans may be used to coordinate sponge behavior. Whereas sensory organs are well-known from ctenophores, in sponges the mechanism for transducing sensory information from the environment has as yet remained unknown.

Here we provide experimental data which suggest that an array of non-motile cilia in the sponge osculum-the chimney-like structure through which water exits from the sponge-functions as a sensory system to detect changes in flow and control whole animal responses. We used an emergent model system, the freshwater sponge, to investigate the ultrastructure and physiology of the cilia. We also studied the molecular evolution of sensory channels of the Transient Receptor Potential family in Porifera. Regardless of whether sponges as we know them today were or were not the earliest multicellular animals to evolve, it is intriguing to consider that an array of sensory cilia like this in sponge oscula could have given rise to more complex signalling cells, such as nerves and sensory sensilla, in the early evolution of animals.

\section{Results and discussion}

\section{Sponge oscula are ciliated}

Sponges are unusual in possessing both cilia and flagella (named for their differing beat patterns [17]) on somatic cells. These include ciliated epithelial cells of sponge larvae which are involved in locomotion and also photoresponses $[18,19]$, ciliated cells at the exit of the feeding choanocyte chambers $[20,21]$ and flagellated choanocytes involved in pumping water through the canal system (reviewed in [20]). In contrast, the epithelia of adult sponges are usually naked. We were therefore surprised to find cilia on all cells forming the epithelial lining of the osculum in the freshwater sponge Ephydatia muelleri, a demosponge that can be cultured in the laboratory (Figure 1a). The osculum is the most prominent feature of a sponge, and is the final exit of water filtered through the sponge body for food and oxygen.

In E. muelleri a pair of cilia, each 4-6 microns long, emerges above the nucleus of every epithelial cell (Figure 1b-f). A survey of 6 other demosponges showed that in each, the oscula are also lined by ciliated cells; in some species the cells have a single cilium, and others up to 4 cilia, all arising centrally above the cell nucleus (Additional file 1: Figure S1). Even glass sponges (class
Hexactinellida), which are syncytial, have cilia at the lip of their large oscula. There is no data available so far for the other two classes, Calcarea and Homoscleromorpha, although the latter is known to have cilia throughout the canals, and therefore presumably also up to the osculum lip.

Serial sections through the base of the cilium in $E$. muelleri show basal bodies are simple, with no structures linking pairs of cilia in a cell (Figure 2a). In contrast to the flagella of choanocyte chambers, which have a central pair of microtubules, in cross section the oscula cilia have a $9+0$ axonemal skeleton (Figure $2 b$ ), which is characteristic of sensory cilia in other organisms [3]. Both fluorescence and scanning electron microscopy show pairs of cilia in E. muelleri are oriented perpendicular to the water flow (Figure 2c), which may be important for sensing changes in flow. In live animals the cilia label with the vital dye FM 1-43, and high frequency time-lapse microscopy showed that they are non-motile and only vibrate in the flow that passes out of the osculum (Figure 2d, and Additional file 2: Movie S1).

\section{Cationic channel blockers inhibit sponge behaviour}

In the last decade it has been recognized that most cells in the vertebrate body, and many in invertebrates, possess specialized sensory structures called 'primary' cilia, which function as sensory organelles as in kidney epithelial cells, chondrocytes, odontoblasts, embryonic endocardial cells, and 'Kupffer's vesicle' [22]. Primary cilia, although similar to motile cilia in their basic structure, lack the radial spokes and dynein arms that enable motility. Instead they possess stretch-activated cationic channels that are part of the transient receptor potential (TRP) channel superfamily [23] including polycystin-1 (PC1) and polycystin-2 (PC2) [23] or their homologs, which allow them to function as sensory organelles [3,22-24]. Remarkably, TRP channels are responsible for almost all forms of sensation experienced by eukaryotic cells, including movement, taste, smell, temperature, vision and osmolarity.

The function of TRP channel sensation is difficult to assess directly, and is therefore usually done by behavioral assay; for example inhibition of an avoidance reaction by the unicellular alga Chlamydomonas using TRP channel blockers has shown that TRP11 is involved in mechanosensation [2]. In multicellular organisms it is difficult to study the function of primary cilia in living tissues, except in cell culture. In contrast, freshwater sponges are small and transparent, and cilia can be viewed live. Furthermore, both of the freshwater sponges E. muelleri and S. lacustris can be triggered to inflate and then contract their whole body (a behaviour termed a 'sneeze' $[14,15]$ ) in response to mechanical or chemical stimuli (Figure 3a). Because the osculum is the final channel through which water exits the sponge, we hypothesized that the cilia have a sensory 


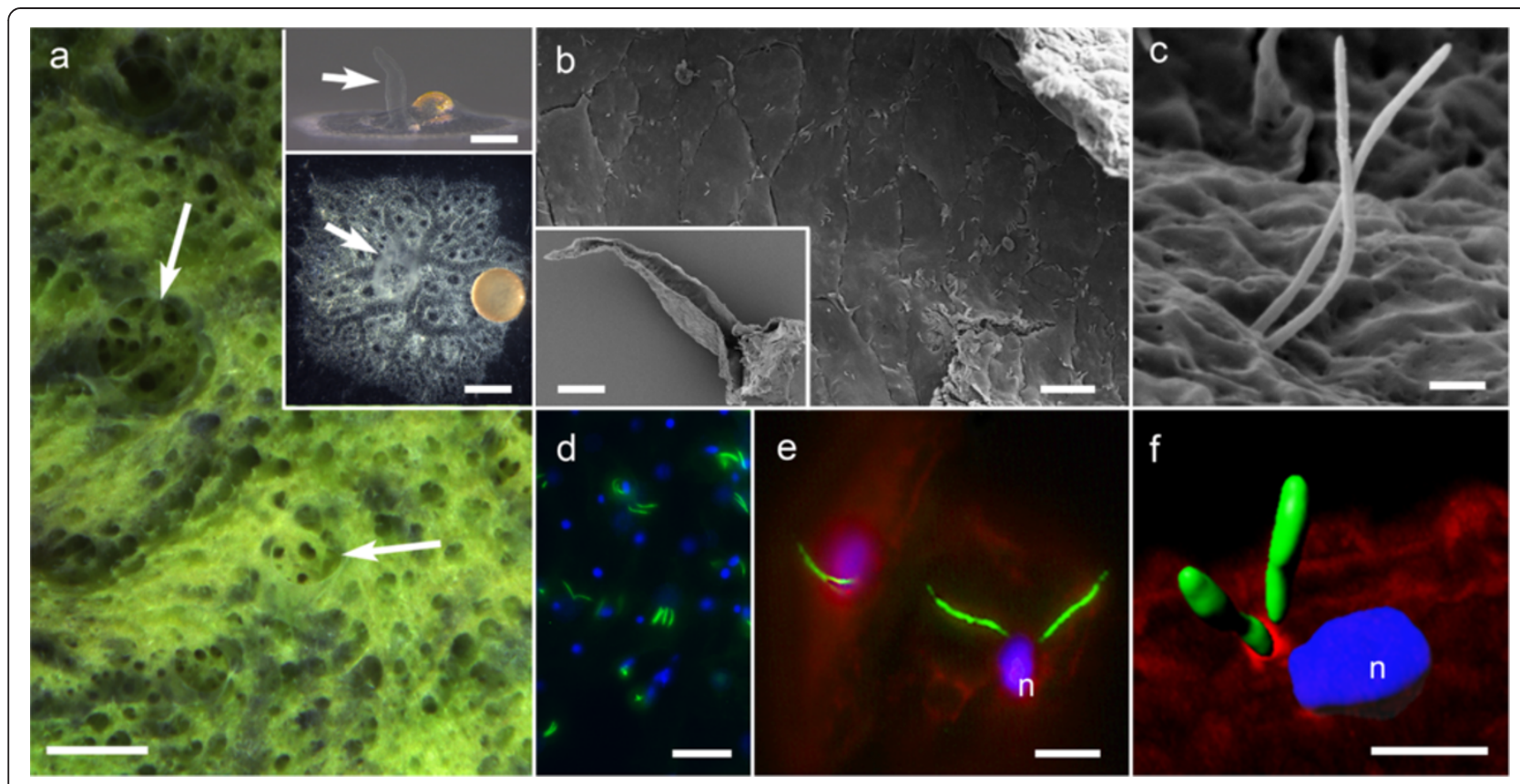

Figure 1 Cilia are found on the epithelia lining the osculum. a. The sponge Ephydatia muelleri in the lake, and grown in the lab viewed from the side (upper inset) and from above (lower inset). The oscula (white arrows) extend upwards from the body. b, c, Scanning electron micrographs show cilia arise from the middle of each cell along the entire length of the inside of the osculum; $\mathbf{b}$ the lining of the osculum with cilia on each cell (inset shows an osculum removed from the sponge and sliced in half longitudinally); c, two cilia arise from each cell. d, e, Cilia in the oscula labeled with antibodies to acetylated a-tubulin (green), nuclei with Hoechst (blue, n), actin with phalloidin (red). f. A 3D surface rendering illustrates how the cilia arise just above the nucleus of the cell. Scale bars a $5 \mathrm{~mm}$; inset $1 \mathrm{~mm} ; \mathbf{b} 20 \mu \mathrm{m}$; inset $100 \mu \mathrm{m} \mathbf{c}, 1 \mu \mathrm{m} \mathbf{d}, 20 \mu \mathrm{m}$ e, f $5 \mu \mathrm{m}$.

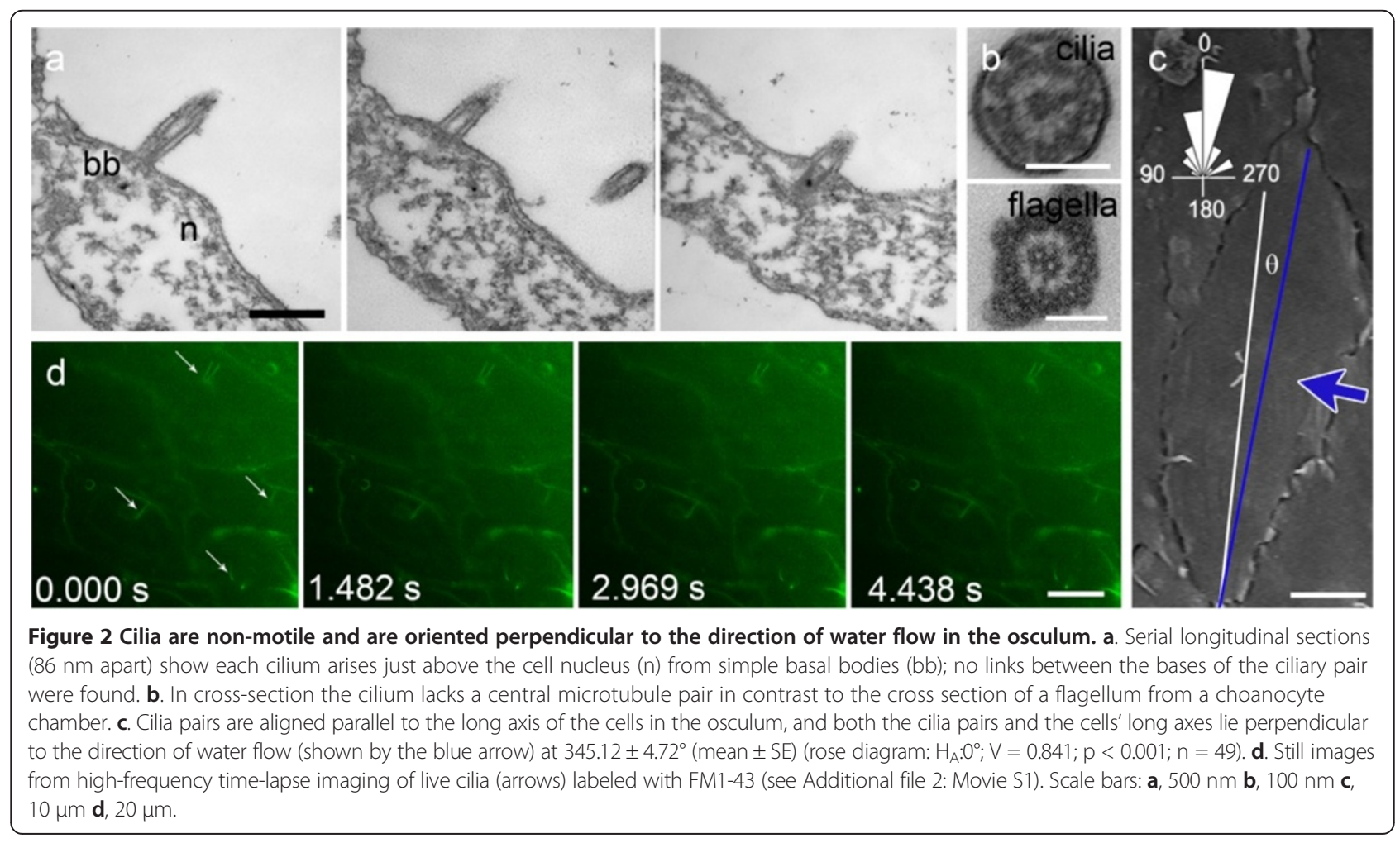




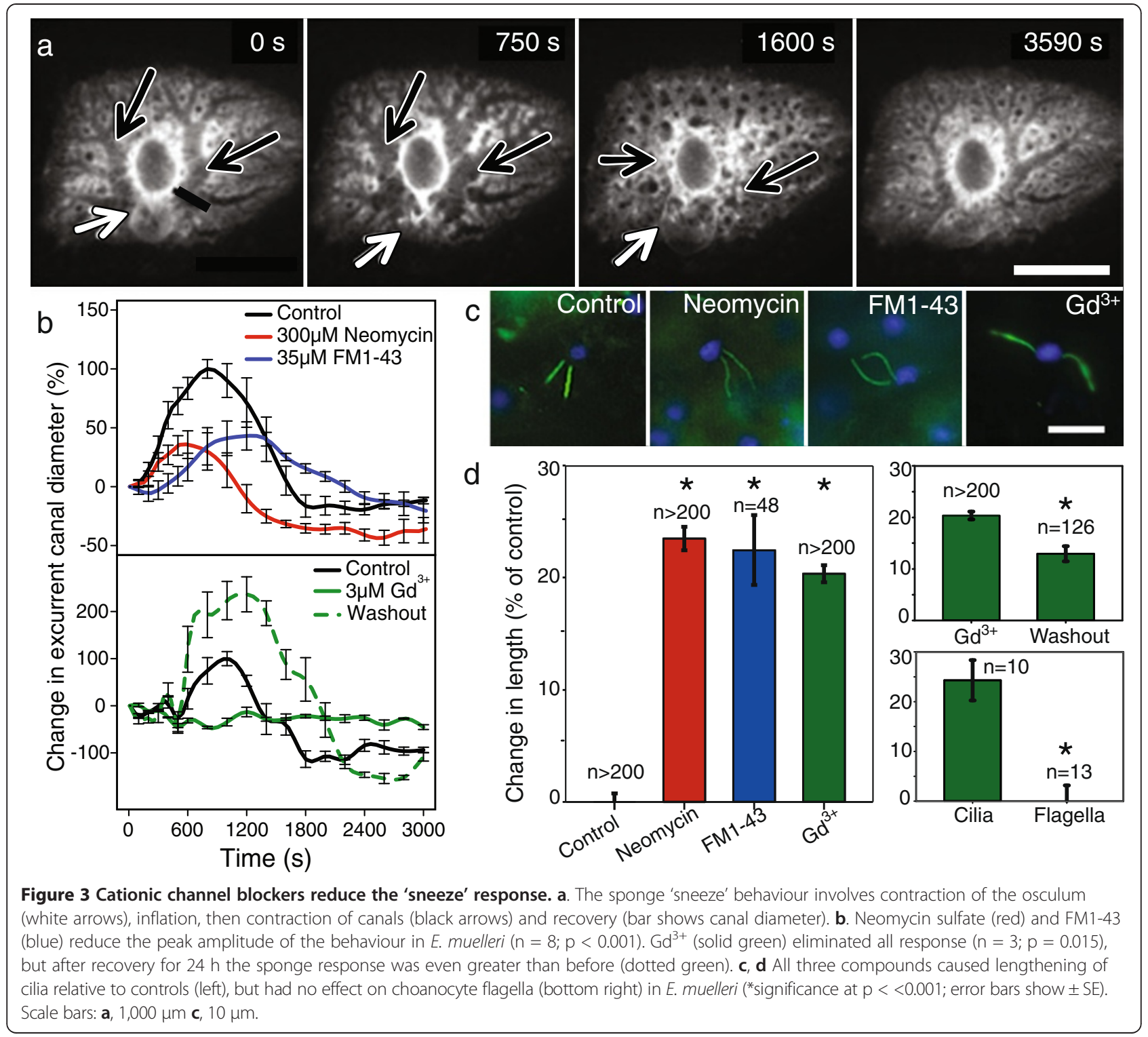

role in controlling the canal diameter to optimize normal flow through the sponge filter, and in particular during the sneeze behaviour.

Three commonly used chemicals-the antibiotic neomycin sulfate, styryl dye FM1-43, and cationic channel blocker Gadolinium $\left(\mathrm{Gd}^{3+}\right)$-have been shown to inhibit sensory ability of primary cilia in other organisms $[25,26]$. These drugs are all thought to block TRPP2 (PC2) channels on the ciliary membrane. In sponges natural stimuli (sediment, vigorous mechanical agitation) as well as bath treatments of 75-90 $\mu \mathrm{M} \mathrm{L-glutamate}$ trigger the inflation and contraction of the excurrent canals $[14,15]$. Treatment of sponges with neomycin sulfate $(300 \mu \mathrm{M})$ and FM 1-43 $(35 \mu \mathrm{M})$ reduced the maximum amplitude of the inflation response by $60 \%$ (Figure $3 \mathrm{~b}$ ) in both cases, and treatment with $\mathrm{Gd}^{3+}(5 \mu \mathrm{M})$ eliminated the response; the effects were reversible (Figure 3b). After recovery, the $\mathrm{Gd}^{3+}$-treated sponges showed an enhanced response to L-Glu (Figure $3 b$ ). This knock-down and knockout of the sponge behaviour by drugs that are known to affect channels on ciliary membranes implicates the cilia in sensing stimuli and transducing them into behaviour. Further support for this idea comes from the direct effect the drugs had on ciliary length.

Lengthening of primary cilia in other organisms has been proposed to increase their sensitivity $[27,28]$. Ciliary (and flagellar) length is determined by a dynamic process of intraflagellar transport (IFT) which continuously brings molecules, including tubulin, up and down the cilium [29]. Chemical or mechanical stimuli that interfere with $\mathrm{Ca}^{2+}$ influx have been shown to alter IFT, thereby changing cilium length $[27,28]$. In $E$. muelleri cilia length increased 
1.2-fold after only one hour of treatment in all three drugs (Figure 3c, d), and $\mathrm{Gd}^{3+}$ treated sponges recovered partially after a one-hour washout. These data suggest that the drugs interfere with IFT in the oscula cilia. Unlike cilia, the flagella in choanocyte chambers of E. muelleri did not change length (Figure 3d), implying that the effects of the drugs reported here are only on ciliated cells.

Although pharmacology is almost universally used to study the sensory roles of cilia and flagella in other organisms [2,25-27,30], neomycin sulfate, FM 1-43, and Gadolinium can also affect other calcium transport processes in tissues including smooth muscle contractility. We therefore tested whether another calcium channel blocker could equally affect the sponges. In contrast to neomycin sulfate which eliminates all response in the sponge, the L-type calcium channel blocker Verapamil $(10 \mu \mathrm{M})$ had no effect on the amplitude of the sneeze reflex (Figure 4a). This finding is consistent with experiments on vertebrate primary cilia $[25,26]$. We found that longer incubation in Neomycin sulfate ( $2 \mathrm{hr}$ in S. lacustris compared to $10 \mathrm{~min}$ for $E$. muelleri) repressed the sneeze reflex for longer. FM 1-43 is fluorescent and was clearly localized primarily to the cilia (Additional file 2: Movie S1), but to determine where neomycin sulfate localized we incubated sponges in Texas Red-conjugates of neomycin sulfate. Cells in the sponge osculum labeled within 2 minutes of incubation in the dye, and the same cells co-labelled with YO-PRO1, which selectively labels hair cells in the lateral line of zebrafish (Danio rerio) [31,32] (Figure 4b). Taken together, the effect of these treatments suggests that stretch-activated, nonselective cation channels are involved in the sponge behavior.

While we cannot rule out the possibility that any of these drugs have other effects on the sponge in addition
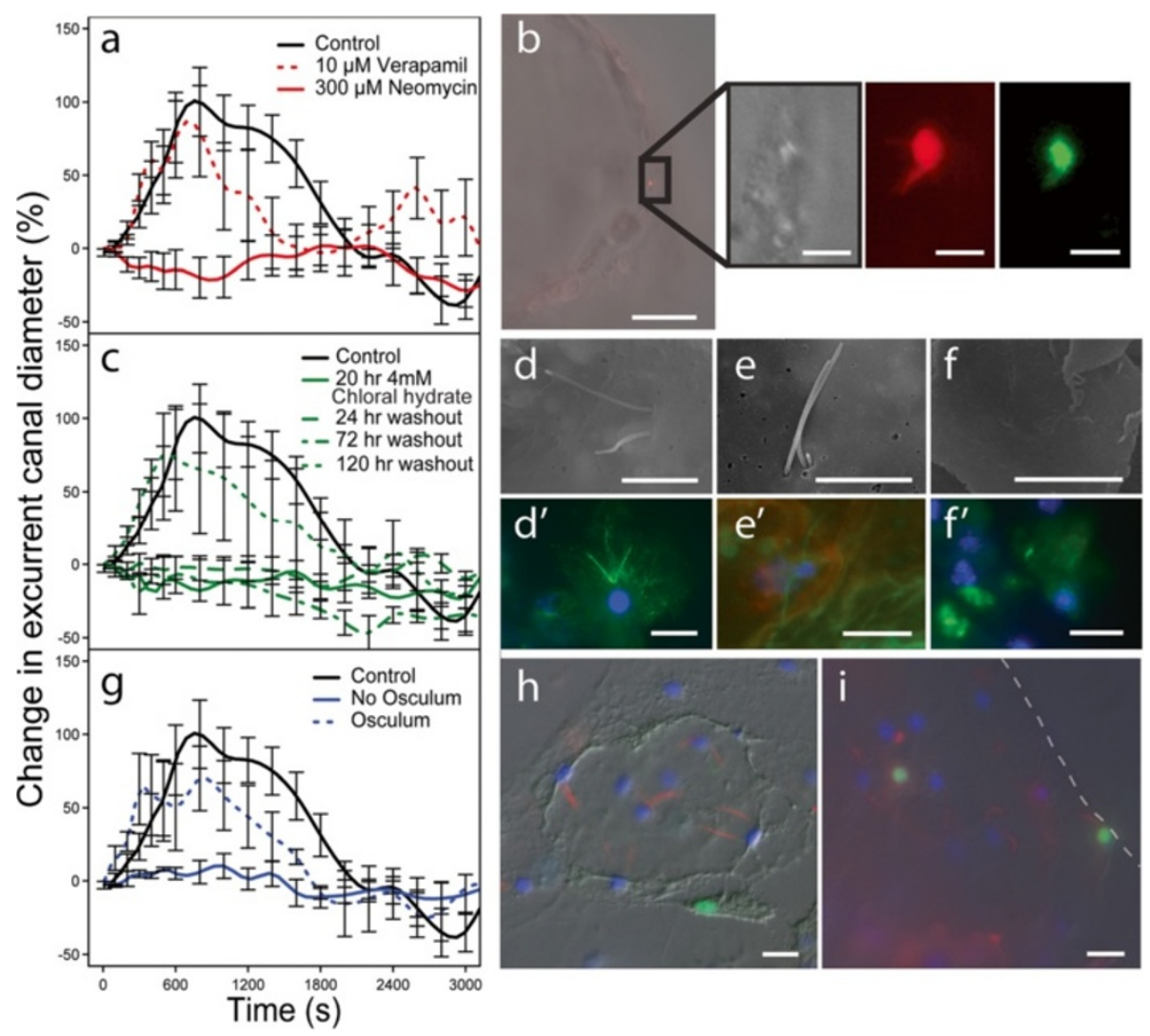

Figure 4 Cilia are specifically involved in the sponge behaviour. a. In contrast to Neomycin sulfate (solid red) which eliminates the 'sneeze' response $(n=3, p=0.035)$, the calcium channel blocker Verapamil (dotted red) does not affect amplitude of the sneeze behaviour in $S$. lacustris $(n=5, p=0.573)$. b. Texas-Red Neomycin sulfate conjugate (red) and YO-PRO1 (green) selectively label cells in the osculum. c. A 20 hr treatment in chloral hydrate eliminates the sneeze behaviour in $S$. lacustris (solid green; $n=5, p=0.004$ ), which does not return until more than 3 days after recovery (dotted green; $n=5,24 \mathrm{hr}$ washout $p=0.003,72 \mathrm{hr}$ washout $p=0.018,120 \mathrm{hr}$ washout $p=0.864)$ ). $\mathbf{d}-\mathbf{f}(\mathbf{S E M}) \mathbf{d}^{\prime}-\mathbf{f}^{\prime}($ fluorescence). Cilia are removed by chloral hydrate treatment; $\mathrm{S}$. lacustris $0 \mathrm{hr}\left(\mathbf{d}, \mathbf{d}^{\prime}\right), 20 \mathrm{hr}\left(\mathbf{e}, \mathbf{e}^{\prime}\right)$, and $70 \mathrm{hr}\left(\mathbf{f}, \mathbf{f}^{\prime}\right)$ treatment in chloral hydrate. $\mathbf{g}$. The sneeze behaviour in S. lacustris cannot be triggered when the osculum is removed (solid blue; $n=3, p=0.010$ ) until it has fully regrown (dotted blue; $n=3, p=0.275$ ). $\mathbf{h}$. Ciliated cells on the surface of $E$. muelleri 8 hr post osculum removal and (i) in the newly formed osculum $24 \mathrm{hr}$ post osculum removal. Ciliated cells do not become labeled with EdU until after the osculum has regrown suggesting they arise by migration of newly formed mesohyl cells which differentiate into ciliated pinacocytes. Cilia are labeled with acetylated a-tubulin (red), nuclei with Hoechst (blue), and newly synthesized DNA with EdU (green). Scale bars: b, $50 \mu \mathrm{m}$ inset $10 \mu \mathrm{m}$ $\mathbf{d}, \mathbf{e}, 5 \mu \mathrm{m} \mathbf{d}^{\prime}, \mathbf{e}^{\prime}, \mathbf{f}, \mathbf{f}^{\prime}, \mathbf{h}, \mathbf{i} 10 \mu \mathrm{m}$. 
to working on the cilia, in our experience very few molecules cause the sponge to relax-most trigger contractions $[15,16]$. However, to confirm that the cilia in the osculum, and the osculum itself, are indeed required for the sponge sneeze reflex we used both chloral hydrate to deciliate the sponge and removed the osculum, and tested the responsiveness of the sponge in each instance. Chloral hydrate is known to remove cilia from cells, causing a loss of behaviour in both metazoans [24] and unicellular eukaryotes [2,4] after $20 \mathrm{hr}$ exposure. It is thought to act by weakening the attachment of the cilium to the basal body, with full loss of cilia occurring after $68 \mathrm{hr}$ in kidney epithelial cells [24]. We found that $20 \mathrm{hr}$ exposure to $4 \mathrm{mM}$ chloral hydrate eliminated the sneeze reflex and it took $120 \mathrm{hr}$ for recovery of sensitivity (Figure 4c-e). As in kidney cells [24], it took $70 \mathrm{hr}$ to remove all cilia from the epithelium of the osculum (Figure 4f).

We have found that when removed, a new osculum forms again after 8 hours. De-osculated sponges could not be triggered to sneeze (Figure 4g), and although the sponge continued to filter water at all times during repair of the osculum, it was only after the osculum had fully formed that the sneeze response returned. Together these results suggest that both the osculum and the cilia lining it are necessary for the sneeze reflex. To determine when ciliated cells first appear on newly formed oscula, we labeled sponges from which the osculum had been removed with the cell proliferation marker EdU and detected incorporation of uridine into new cells using Click-iT (Molecular Probes, Invitrogen). At $8 \mathrm{hr}$ after the osculum was removed, cilia were found on cells in a few discrete places on the surface of the sponge (Figure 4h). Pinacocytes in the sponge surface are not usually ciliated, therefore we interpreted the differentiation of cilia on pinacocytes as an early marker of the location of a new osculum. Furthermore, although mesohyl cells were labeled within $6 \mathrm{hrs}$ of incubation in EdU, cells of the new osculum were not labeled with EdU, and it was only $24 \mathrm{hr}$ after the new osculum was formed that a few new ciliated cells labeled (Figure 4i). Although we were unable to trace the migration of cells in live animals, we interpret these data to suggest that cilia differentiate on cells in the surface of the sponge, thereby identifying the region as a potential osculum; then as the osculum grows to full height using cells already present in the sponge, new ciliated epithelial cells differentiate from newly formed mesohyl cells.

\section{Sponges possess a repertoire of transient receptor potential channels}

Considering the conserved role of TRP channels, and in particular PKD in sensory behaviour across eukaryotes [2], we searched the transcriptomes of 8 sponge species for homologs of both $p k d 1$ and $p k d 2$ and other TRP channels. A 700aa homolog of $p k d 2$ (Type II TRP) was identified in Corticium candelabrum (Homoscleromorpha) and a 178aa sequence of a $p k d 2$ (Type II TRP) gene was found in the freshwater Spongilla lacustris (Demospongiae) (Figure 5a, Additional file 1: Figures S2, S3). We found a 978aa sequence of a Type II TRP (ML) in Sycon coactum (Calcarea), and several sequences with similarity to various Type I TRP channels were found in all 4 Porifera classes (Figure 5a-c, Additional file 1: Figure S5). These candidates were included in an alignment containing more than 100 representatives for all the TRP families across bilaterians (Figure 5a; Additional file 1: Figures S2-S3). The ability to retrieve protein sequences depends on the quality of the transcriptome and the divergence of sequences in transcriptomes. Negative results do not imply conclusive absence. Our phylogenetic analysis grouped sponge $p k d$ sequences with Type II TRP and specifically $p k d 2$ channels genes from bilaterians with high support (91\% bootstrap). Sponge $p k d$ channel sequences showed similar domain architecture and proposed 3D protein folding to both mouse and Chlamydomonas sequences (Figure 5b), and other sponge sequences showed amino acids indicative of the TRP domain (Figure 5c; Additional file 1: Figure S5). Although the pharmacology of the sponge cilia is similar to that of cilia known to have $p k d 2$ channels, several TRP channels from Chlamydomonas have also been found to transduce mechanical signals so we cannot rule out the possibility that other TRP channels are involved in flow sensing in sponges.

\section{Conclusions}

In the sponge, obstruction of the canals by particulates in the feeding current would cause changes in pressure across the system; the osculum is the single exit of the entire system and is expected to be sensitive to this change, so it is plausible that the cilia detect changes in water flow or pressure. The absence of motility of the cilia, and their specific localization to the inner lining of the sponge osculum strongly suggest a sensory role for the osculum; the pharmacology and ablation experiments also support the hypothesis that the cilia have a sensory function. The primary cilium, which extends out from the cell and has a high surface-area to volume ratio, is an ideal organelle for both sensing and transducing signals [3]. These cilia in the sponge osculum have all the characteristics of primary cilia.

While the role of cilia in sensing information may have evolved many times within eukaryotes, the sponge sensory system described here is certainly very similar to signalling via primary cilia in other metazoans [22]. The role of cilia in the sponge osculum suggests either a convergent role in sensing and transducing flow information into behaviour across all metazoa, or implies that primary cilia had an ancient evolutionary role in transducing sensory information, and in particular flow, in early multicellular animals. Given 


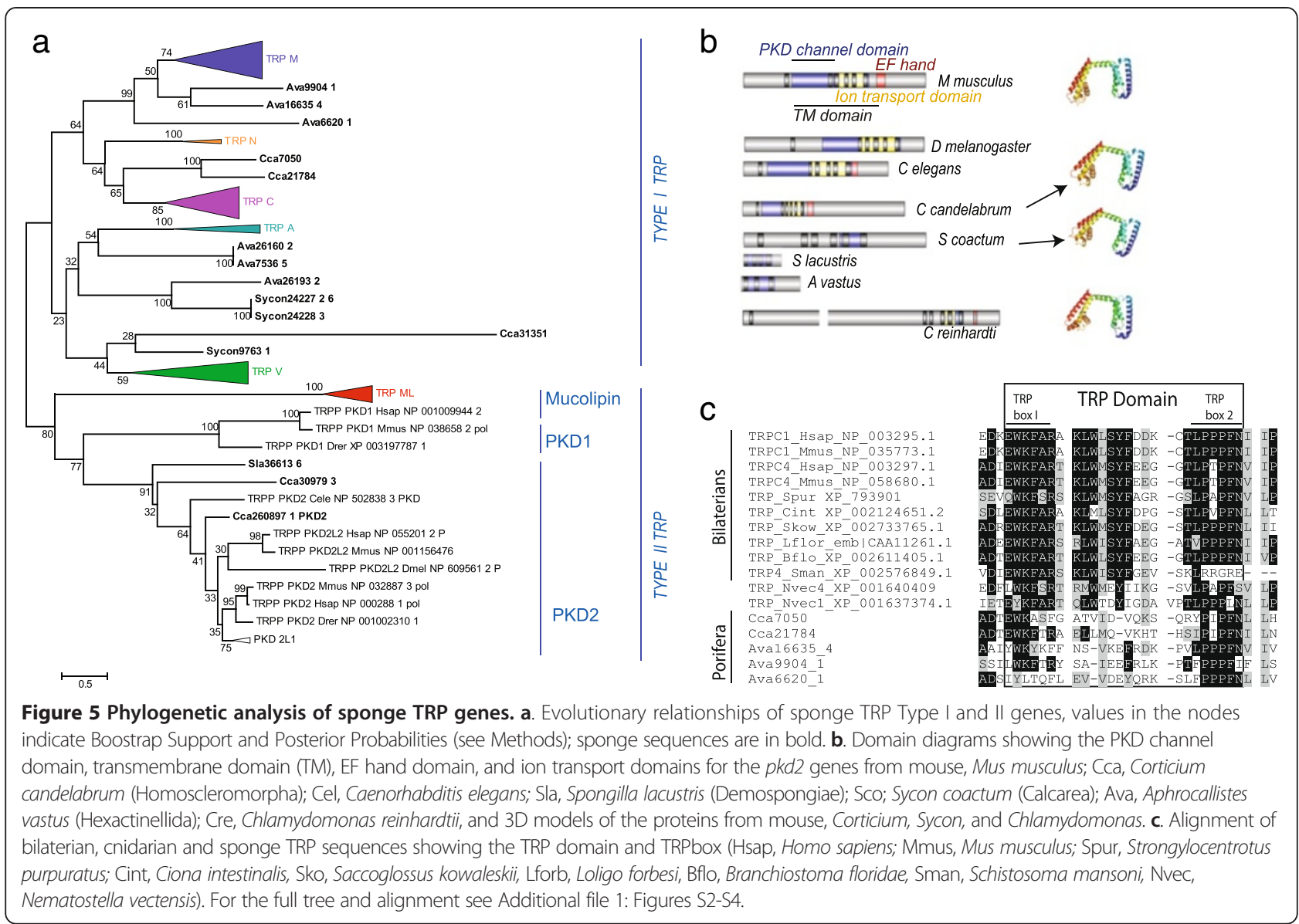

the unique position of Porifera as extant representatives of one of the first groups of multicellular animals [9], and in particular their lack of conventional nervous and coordination systems, the finding of such an organized array of sensory cells in sponges provides new insight into possible mechanisms of evolution of early sensory systems.

\section{Methods}

\section{Collecting and culturing of sponges}

Gemmules of the freshwater sponges Ephydatia muelleri and Spongilla lacustris were collected from Frederick Lake, BC and Rousseau Lake, BC, respectively, at a depth of $0-3 \mathrm{~m}$ and stored at $4^{\circ} \mathrm{C}$ in unfiltered lake water, aerated monthly, until use. The spicule skeleton was removed from the gemmules by gently rubbing between two pieces of corduroy, and the gemmules were then sorted, sterilized (using $1 \% \mathrm{H}_{2} \mathrm{O}_{2}$ for $5 \mathrm{~min}$ ), and rinsed in cold distilled water. Single gemmules placed onto ethanol sterilized glass coverslips in Petri dishes containing Mmedium [33], hatched in 2-3 days; culture medium was changed every $2 d$ post hatching (dph). Only 5-10 dph sponges with fully developed aquiferous canal systems were used in experiments.

\section{Fixation for fluorescence microscopy}

Sponges on coverslips were fixed in $3.7 \%$ paraformaldehyde and $0.3 \%$ glutaraldehyde in $100 \mathrm{mM}$ phosphate-buffered saline (PBS) for $12-24 \mathrm{~h}$ at $4^{\circ} \mathrm{C}$. Preparations were rinsed three times in cold PBS, permeabilized with $\mathrm{PBS}+0.1 \%$ Triton X-100 (PBTX) for two minutes and rinsed in PBS. Either whole juvenile sponges or individual oscula (pulled off of the sponge by pinching the base of the osculum with fine forceps) were labeled with mouse anti-acetylated alpha tubulin (Sigma-Aldrich, Oakville, ON) in 10\% goat serum (GS) and PBS at 1:1000 at RT overnight. Preparations were rinsed in PBS and incubated in goat anti-mouse 488 (Molecular Probes, Burlington, ON) at 1:100 in 10\% GS and PBS overnight. Nuclei were counterstained with Hoescht 33342 (Sigma-Aldrich) 1:100 in PBS for $10 \mathrm{~min}$. Some preparations were stained for actin using Alexa 594 phalloidin (Molecular Probes) in BSA-PBS. Labelled oscula were sliced open using a microscalpel, mounted on a slide in $100 \%$ glycerol, and sealed with nail polish. Whole sponges on coverslips were inverted onto a slide in $100 \%$ glycerol, sealed with nail polish and viewed with a Zeiss Axioskop2 Plus. Confocal images were taken using a Zeiss LSM 710, and surface rendering was done using Imaris v7.2 (Bitplane, Zurich, Switzerland). 


\section{Fixation for scanning and transmission electron microscopy (SEM, TEM)}

Hatched sponges were fixed and prepared for electron microscopy as described previously [14]. For SEM oscula were removed from the sponges, dehydrated to $100 \%$ ethanol and critical point dried. Dried oscula were mounted on aluminum stubs using adhesive tabs and gold-coated prior to viewing using a scanning electron microscope (JEOL $6301 \mathrm{~F}$ field emission or a Zeiss EVO MA 15). For TEM oscula were dehydrated through $100 \%$ ethanol and embedded in epoxy (TAAB 812). Ultrathin sections $(60 \mathrm{~nm})$ were stained with uranyl acetate and lead citrate and viewed in a Hitatchi $\mathrm{H}-7000$ or Phillips Morgagni (FEI) TEM and images captured with an AMT or Gatan digital camera respectively.

\section{Orientation analysis}

To assess orientation of each cilia pair with respect to the direction of water flow along the osculum, a line was drawn between the base of the two cilia and the angle between that line and a line defining the long axis of the cell was calculated using ImageJ (v1.43r; NIH, Bethesda, MD). Circular statistics calculated with Oriana v. 3.13 (KCS, Wales, UK) gave the mean angle of the orientation of cilia pairs and a V-test was performed to determine difference from the long axis of the cell.

\section{Assessment of the possible sensory role}

Stock solutions of $10 \mathrm{mM}$ neomycin sulfate (Fisher BioReagents, New Jersey), 1 g/L (178.5 M) FM 1-43FX (Fixable analog; Molecular probes, Invitrogen), $10 \mathrm{mM}$ of $\mathrm{GdCl}_{3}$ (Sigma-Aldrich), $20 \mathrm{mM}$ Verapamil (Sigma-Aldrich), and $1 \mathrm{M}$ Chloral hydrate (Sigma-Aldrich) were kept covered at $4^{\circ} \mathrm{C}$ and used at $300 \mu \mathrm{M}, 35 \mu \mathrm{M}, 5 \mu \mathrm{M}, 10 \mu \mathrm{M}$, and $4 \mathrm{mM}$ respectively. Neomycin sulfate and FM1-43FX were added to the Petri dish and the sponge was stimulated using agitation (vigorous shaking of the Petri dish for $30 \mathrm{~s}$ ) $10 \mathrm{~min}$ later for E. muelleri or $2 \mathrm{hr}$ later for S. lacustris. $\mathrm{Gd}^{3+}$ and Verapamil were added to the Petri dish $2 \mathrm{hr}$ prior to stimulation with 75-90 $\mu \mathrm{M}$ L-Glutamate. Treatment in chloral hydrate was for $20 \mathrm{hr}$ prior to stimulation with 75-90 $\mu \mathrm{M}$ L-Glutamate; during washout the Mmedium was changed every $2 \mathrm{~d}$ and the sponge was then stimulated with 75-90 $\mu \mathrm{M}$ L-Glutamate. Oscula were removed by pinching the base of the osculum with fine-tipped forceps, and the sponge was stimulated at $2 \mathrm{hr}$ and then again at $24 \mathrm{hr}$ using agitation. Care was taken to add each treatment to the side of the Petri dish away from the sponge, and the solution was mixed by pipetting gently 5-6 times. Images were captured every $10 \mathrm{~s}$ for $50 \mathrm{~min}$, or until the sponge had completed an inflation/contraction cycle. Still images were captured in Northern Eclipse v.7 (Empix Imaging Inc., Mississauga, $\mathrm{ON}$, Canada). Changes in canal diameter were measured every tenth image for the first 60 images, and then every $20^{\text {th }}$ image, using ImageJ (v1.43r; NIH). Due to high variation in changes in canal diameters within a single sponge, three canals in each sponge were measured for the neomycin and FM test and a nested ANOVA was run in $\mathrm{R}$ (v.2.4.1). The variance between canals within a single individual did not account for any of the variance in the dataset, therefore only one canal was measured per sponge in the remaining experiments and tested via a one-way ANOVA in R (v.2.4.1). All data were tested for normality using a Shapiro-Wilks test, with $\mathrm{Gd}^{3+}$ data $\log (\mathrm{x})$ transformed and chloral hydrate data square root transformed.

Cilia length of sponges treated with neomycin sulfate, FM1-43FX and $\mathrm{Gd}^{3+}$, for one hour each, were measured from fluorescence images with ImageJ (v1.43r). Untreated sponges were used as controls. Reversibility of $\mathrm{Gd}^{3+}$ treatment was demonstrated by washing out the blocker for $1 \mathrm{hr}$ in culture medium prior to fixation. Cilia and flagella length of $\mathrm{Gd}^{3+}$-treated sponges were measured from SEM images. The measurements were $\log (\mathrm{x})$ transformed and analyzed using a nested ANOVA in R (v.2.4.1).

Texas-Red conjugated neomycin (TR-Neo) was made by shaking neomycin sulfate $\left(50 \mathrm{mg} / \mathrm{ml}\right.$ in $\left.\mathrm{K}_{2} \mathrm{CO}_{3}\right)$ and Texas Red (Molecular Probes, Invitrogen; $2 \mathrm{mg} / \mathrm{ml}$ in dimethylformamide) overnight [34], and added to M-medium to a final concentration of $300 \mu \mathrm{M}$ neomycin sulfate. S. lacustris was treated for $2 \mathrm{~min}$ in TR-Neo followed by three rinses in M-medium, $5 \mathrm{~min}$ in $1 \mu \mathrm{M}$ YO-PRO1 (Invitrogen) [31,32], and three more rinses in M-medium prior to viewing live using a 40X Zeiss water immersion objective.

Both whole $S$. lacustris and oscula removed from the sponge were treated in $4 \mathrm{mM}$ chloral hydrate for $20 \mathrm{hr}$ or $70 \mathrm{hr}$ (medium changed daily to maintain concentration), and fixed for fluorescence microscopy with anti-tubulin and for SEM as described above. Click-iT EdU imaging kit (Invitrogen) was used to label newly synthesized cells post osculum removal. E. muelleri was incubated in $50 \mu \mathrm{M}$ EdU in M-medium for $8 \mathrm{hr}$ or $24 \mathrm{hr}$ after the osculum was removed, fixed for fluorescence, and labeled using the Click copper-catalyzed covalent reaction. Sponges were labelled with acetylated alpha tubulin and Hoechst as described above.

\section{Biolnformatics}

The transcriptomes of 8 sponge species (Ephydatia muelleri, Spongilla lacustris, Petrosia ficiformis, Chondrilla nucula, Ircinia fasciculata, Corticium candelabrum, Sycon coactum, Aphrocallistes vastus) were sequenced using Illumina and assembled de novo in either Trinity or CLCGenomics Workbench 5.1 [35]. TRP sequences in these transcriptomes and also in the Amphimedon queenslandica genome [36] were detected using HMMer (Janelia.org) using HMM profiles formed with $p k d 1$ and $p k d 2$ sequences 
collected from NCBI or by blasting NCBI sequences against the transcriptome datasets using the tblastn suite in CLC Genomics Workbench. Sequence identity and domain conservation was confirmed by BLAST and NCBI's conserved domain search as well as EMBL's InterPro Scan; domain illustrations were conceived using DOG2.0 and 3D models projected using Phyre2 [37]. TRP channel and PKD channel sequences from bilaterians were downloaded from SwissProt following the (vertebrates) taxon sampling for TRP and PKD domains in Pfam [38]; SwissProts accession numbers are indicated in the sequence labels. Chlamydomonas reinhardti PKD2 ABR14113.1 was downloaded from NCBI. For phylogenetic analysis sequences were aligned in MAFFT [39] using the E-INSI algorithm, and positions shared by $85 \%$ of the taxa were selected using MEGA5.1 [40] for further phylogenetic analyses. Evolutionary relationships were inferred by ML using the evolutionary model LG [41] + GAMMA + Invariants as implemented in RAxML [42]. The statistical support of the branches was obtained by generating 1000 bootstrap pseudoreplicates. (The full alignment of 344aa and tree are shown in Additional file 1: Figures S2, S3.) The same dataset was analyzed under the Bayesian Inference framework/Phylobayes-MPI [43] under the CAT-GTR [44] model (Additional file 1: Figure S4). The tree search was conducted during 7,500 cycles, and a burning of 1000 trees (sub-sampling every 10 trees) was used to discard the trees before the search reached the likelihood optima.

\section{Availability of supporting data}

Full alignments and trees are provided in supplemental data files. Sponge sequences described here have been deposited at DDBJ/EMBL/GenBank under the BioProjects PRJNA162903, PRJNA225591, PRJNA162899, PRJNA225584.

\section{Additional files}

Additional file 1: Figure S1. Cilia in the oscula of various demosponges a. Ephydatia muelleri,b, c. Spongilla lacustris, d. Neopetrosia vanilla, e. Haliclona mollis, f. Haliclona sp. g. Neopetrosia problematica, h. Aphrocallistes vastus. Scale bars 1 m. Figure S2: Uncompressed tree showing the evolutionary relationships of sponge TRP Type I and II genes. Values at nodes indicate Bootstrap support. Figure S3: Full alignment of TRP sequences for the uncompressed tree from Figure 5a. Figure S4: Phylobayes alignment of data in 5c. Figure S5: Full alignment of sequences in Figure $5 \mathrm{C}$ and list of Sponge TRP Fastas.

Additional file 2: Movie S1. Cilia in the osculum of a live sponge, Ephydatia muelleri, labeled using FM1-43. High-frequency time-lapse microscopy (images taken at 50 millisecond intervals with exposure of 50 milliseconds) indicates that the cilia are non-motile and only vibrate in the flow that passes out the osculum.

\section{Competing interests}

The authors declare that they have no competing interests.

\section{Authors' contributions}

DAL and SPL conceived the experiments; SPL collected the sponges; DAL performed the experiments and carried out the statistical analysis; DAL and SPL carried out the electron microscopy; NF, AR, SPL and JP performed the molecular analysis; DAL and SPL wrote the paper. All authors read and acknowledged the final version of the manuscript.

\section{Acknowledgements}

We thank AR Palmer, WT Allison, X-Z Chen, A Kahn, and PJ Windsor-Reid for comments on an earlier version of this manuscript. X Sun (Cross Cancer Institute, U Alberta) and B Gowan (UVic Biology Department EM Lab) helped with Confocal and Transmission Electron microscopy respectively. EDM Adams provided early guidance with culturing and tissue manipulations. Image 1a was taken by $\mathrm{N}$ Lauzon, 1a (upper inset) by GRD Elliott, and S1h by A Kahn. This work was funded by an NSERC Discovery Grant to SPL.

\section{Author details}

${ }^{1}$ Department of Biological Sciences, University of Alberta, CW 405 Biological Sciences Building, Edmonton, Alberta T6G 2E9, Canada. ${ }^{2}$ Department of Animal Biology, Universitat de Barcelona, Avinguda Diagonal 643, Barcelona 08028, Spain. ' ${ }^{3}$ Department of Zoology, University of Oxford, Oxford OX1 3PS, UK.

Received: 4 September 2013 Accepted: 11 December 2013

Published: 13 January 2014

\section{References}

1. Ryan TJ, Grant SGN: The origin and evolution of synapses. Nat Rev Neurosci 2009, 10:701-712.

2. Fujiu K, Nakayama Y, lida H, Sokabe M, Yoshimura K: Mechanoreception in motile flagella of Chlamydomonas. Nat Cell Biol 2011, 13:630-632.

3. Singla V, Reiter JF: The primary cilium as the cell's antenna: signaling at a sensory organelle. Science 2006, 313:629-633.

4. Dunlap K: Localization of calcium channels in Paramecium caudatum. J Physiol 1977, 271:119-133.

5. Meech RW: Non-neural reflexes: sponges and the origins of behaviour. Curr Biol 2008, 18:R70-R72.

6. Mackie GO: Neuroid conduction and the evolution of conducting tissues. Q Rev Biol 1970, 45:319-332.

7. Sperling EA, Peterson KJ, Pisani D: Phylogenetic-signal dissection of nuclear housekeeping genes supports the paraphyly of sponges and the monophyly of Eumetazoa. Mol Biol Evol 2009, 26:2261-2274.

8. Philippe H, Derelle R, Lopez P, Pick K, Borchiellini C, Boury-Esnault N, Vacelet J, Renard E, Houliston E, Quéinnec E, et al: Phylogenomics revives traditional views on deep animal relationships. Curr Bio/ 2009, 19:706-712.

9. Roure $B$, Baurain $D$, Philippe $H$ : Impact of missing data on phylogenies inferred from empirical phylogenomic data sets. Mol Biol Evol 2013, 30:197-214.

10. Nosenko T, Schreiber F, Adamska M, Adamski M, Eitel M, Hammel J, Maldonado M, Müller WEG, Nickel M, Schierwater B, et al: Deep metazoan phylogeny: when different genes tell different stories. Mol Phylogenet Evol 2013, 67:223-233.

11. Dunn CW, Hejnol A, Matus DQ, Pang K, Browne WE, Smith SA, Seaver E, Rouse GW, Obst M, Edgecombe GD, et al: Broad phylogenomic sampling improves resolution of the animal tree of life. Nature 2008, 452:745-749.

12. Ryan JF, Pang K, Schnitzler CE, Nguyen A-D, Moreland T, Simmons DK, Koch BJ, Francis WR, Havlak P, Comparative Sequencing Program NISC, Smith SA, Putnam NH, Haddock SHD, Dunn CW, Wolfsberg TG, Mullikin JC, Martindale $M Q$, Baxevanis AD: The genome of the ctenophore Mnemiopsis leidyi and its implications for cell type evolution. Science 2013, 342:1336-1340.

13. Conaco C, Bassett DS, Zhou H, Arcila ML, Degnan SM, Degnan BM, Kosik KS: Functionalization of a protosynaptic gene expression network. Proc Natl Acad Sci U S A 2012, 109(Suppl)):10612-10618.

14. Sakarya O, Armstrong K, Adamska M, Adamski M, Wang I-F, Tidor B, Degnan BM, Oakley TH, Kosik KS: A post-synaptic scaffold at the origin of the animal kingdom. PloS one 2007, 2:e506.

15. Elliott GRD, Leys SP: Coordinated contractions effectively expel water from the aquiferous system of a freshwater sponge. J Exp Biol 2007, 210:3736-3748. 
16. Elliott GRD, Leys SP: Evidence for glutamate, GABA and NO in coordinating behaviour in the sponge, Ephydatia muelleri (Demospongiae, Spongillidae). J Exp Biol 2010, 213:2310-2321.

17. Linck RW: Chemical and structural differences between cilia and flagella from the lamellibranch mollusc, Aequipecten irradians. J Cell Sci 1973, 12:951-981.

18. Leys SP, Degnan BM: Embryogenesis and metamorphosis in a haplosclerid demosponge: gastrulation and transdifferentiation of larval ciliated cells to choanocytes. Invertebr Biol 2002, 121:171-189.

19. Rivera AS, Ozturk N, Fahey B, Plachetzki DC, Degnan BM, Sancar A, Oakley $\mathrm{TH}$ : Blue-light-receptive cryptochrome is expressed in a sponge eye lacking neurons and opsin. J Exp Biol 2012, 215:1278-1286.

20. Leys SP, Hill A: The physiology and molecular biology of sponge tissues. Adv Mar Biol 2012, 62:1-56.

21. De Vos L, Boury-Esnault N, Vacelet J: The apopylar cells of sponges. In New Perspectives in Sponge Biology; 1990:153-158.

22. Praetorius HA, Spring KR: A physiological view of the primary cilium. Annu Rev Physiol 2005, 67:515-529.

23. Nauli SM, Alenghat FJ, Luo Y, Williams E, Vassilev P, Li X, Elia AEH, Lu W, Brown EM, Quinn SJ, et al: Polycystins 1 and 2 mediate mechanosensation in the primary cilium of kidney cells. Nat Genet 2003, 33:129-137.

24. Praetorius HA, Spring KR: Removal of the MDCK cell primary cilium abolishes flow sensing. J Membr Biol 2003, 191:69-76.

25. Gale JE, Marcotti W, Kennedy HJ, Kros CJ, Richardson GP: FM1-43 dye behaves as a permeant blocker of the hair-cell mechanotransducer channel. J Neurosci 2001, 21:7013-7025.

26. Praetorius HA, Spring KR: Bending the MDCK cell primary cilium increases intracellular calcium. J Membr Biol 2001, 184:71-79.

27. Besschetnova TY, Kolpakova-Hart E, Guan Y, Zhou J, Olsen BR, Shah JV: Identification of signaling pathways regulating primary cilium length and flow-mediated adaptation. Curr Biol 2010, 20:182-187.

28. Miyoshi K, Kasahara K, Miyazaki I, Asanuma M: Factors that influence primary cilium length. Acta Med Okayama 2011, 65:279-285.

29. Rosenbaum JL, Witman GB: Intraflagellar transport. Nat Rev Mol Cell Biol 2002, 3:813-825.

30. Harris JA, Cheng AG, Cunningham LL, MacDonald G, Raible DW, Rubel EW: Neomycin-induced hair cell death and rapid regeneration in the lateral line of zebrafish (Danio rerio). J Assoc Res Otolaryngol 2003, 4:219-234.

31. Santos F, MacDonald G, Rubel EW, Raible DW: Lateral line hair cell maturation is a determinant of aminoglycoside susceptibility in zebrafish (Danio rerio). Hear Res 2006, 213:25-33.

32. Ou HC, Cunningham LL, Francis SP, Brandon CS, Simon J, Raible DW, Rubel EW: Identification of FDA-approved drugs and bioactives that protect hair cells in the zebrafish (Danio rerio) lateral line and mouse (Mus musculus) utricle. J Assoc Res Otolaryngol 2009, 10:191-203.

33. Rasmont R: Une technique de culture des eponges d'éau douce en milieu controle. Ann Soc R Zool Belg 1961, 91:149-155.

34. Steyger PS, Peters SL, Rehling J, Hordichok A, Dai CF: Uptake of gentamicin by bullfrog saccular hair cells in vitro. J Assoc Res Otolaryngol 2003 4:565-578.

35. Riesgo A, Pérez-Porro AR, Carmona S, Leys SP, Giribet G: Optimization of preservation and storage time of sponge tissues to obtain quality mRNA for next-generation sequencing. Mol Ecol Resour 2012, 12:312-322.

36. Srivastava M, Simakov O, Chapman J, Fahey B, Gauthier M, Mitros T, Richards GS, Conaco C, Dacre M, Hellsten U, et al: The Amphimedon queenslandica genome and the evolution of animal complexity. Nature 2010, 466(7307):720-727.

37. Kelley LA, Sternberg MJE: Protein structure prediction on the Web: a case study using the Phyre server. Nat Protoc 2009, 4:363-371.

38. Punta M, Coggill PC, Eberhardt RY, Mistry J, Tate J, Boursnell C, Pang N Forslund K, Ceric G, Clements J, et al: The Pfam protein families database. Nucleic Acids Res 2012, 40:D290-D301.

39. Katoh K, Misawa K, Kuma Kl, Miyata T: MAFFT: a novel method for rapid multiple sequence alignment based on fast Fourier transform. Nucleic Acids Res 2002, 30:3059-3066.

40. Tamura K, Peterson D, Peterson N, Stecher G, Nei M, Kumar S: MEGA5: molecular evolutionary genetics analysis using maximum likelihood, evolutionary distance, and maximum parsimony methods. Mol Biol Evol 2011, 28:2731-2739.

41. Le SQ, Gascuel O: An improved general amino acid replacement matrix. Mol Biol Evol 2008, 25:1307-1320.
42. Stamatakis A: RAxML-VI-HPC: maximum likelihood-based phylogenetic analyses with thousands of taxa and mixed models. Bioinformatics 2006, 22:2688-2690

43. Lartillot N, Rodrigue N, Stubbs D, Richer J: PhyloBayes MPI: phylogenetic reconstruction with infinite mixtures of profiles in a parallel environment. Syst Biol 2013, 62(4):611-615.

44. Lartillot N, Philippe $\mathrm{H}$ : A Bayesian mixture model for across-site heterogeneities in the amino-acid replacement process. Mol Biol Evol 2004, 21:1095-1109.

doi:10.1186/1471-2148-14-3

Cite this article as: Ludeman et al:: Evolutionary origins of sensation in metazoans: functional evidence for a new sensory organ in sponges. BMC Evolutionary Biology 2014 14:3.

\section{Submit your next manuscript to BioMed Central and take full advantage of:}

- Convenient online submission

- Thorough peer review

- No space constraints or color figure charges

- Immediate publication on acceptance

- Inclusion in PubMed, CAS, Scopus and Google Scholar

- Research which is freely available for redistribution 